Neurosurg Focus 19 (5):E7, 2005

\title{
Modeling medulloblastoma with genetically engineered mice
}

\author{
DANiEl W. Fults, M.D. \\ Department of Neurosurgery, University of Utah School of Medicine, Salt Lake City, Utah
}

\begin{abstract}
Medulloblastoma is a malignant tumor that arises in the cerebellum in children, presumably by transformation of granule neuron precursor cells. In vivo models of medulloblastoma in genetically engineered mice have shown that activation of signal transduction pathways that stimulate proliferation and inhibit differentiation of neural progenitor cells during cerebellar development initiate medulloblastoma formation. Activation of the Sonic hedgehog (Shh)/Patched signaling pathway in the postnatal cerebellum is sufficient to induce medulloblastoma in mice. Activation of the phosphatidylinositol 3-kinase (PI3K) signaling pathway by insulin-like growth factor-II, inactivation of the p53 tumor suppressor protein, loss of DNA damage repair mechanisms, and ectopic expression of Myc oncoproteins cooperate with $\mathrm{Shh} /$ Patched signaling to enhance tumor formation in mice. Ectopic expression of alpha and beta interferons in the developing brain also induces Shh-mediated medulloblastoma formation, suggesting a possible role for antiviral response in the genesis of medulloblastoma. By revealing which cell signaling proteins can initiate medulloblastoma formation, mouse models have enabled investigators to identify molecular targets for designing new therapies. Small-molecule inhibitors of the Shh/Patched and PI3K pathways are potential chemotherapeutic agents for patients with medulloblastoma.
\end{abstract}

\section{KEY WORDS • medulloblastoma • Sonic hedgehog gene • phosphatidylinositol 3-kinase • signal transduction - mouse}

\section{OVERVIEW}

Over the past several decades, rapid expansion in our understanding of the molecular biology of cancer has dispelled any hope that this disease might be explained by mutations in a small number of genes. On the contrary, cancer cells have many mutant genes and dysfunctional proteins. Although this fact may pique the interest of basic scientists who challenge themselves by solving complex problems, it makes it difficult for clinical scientists to select targets for new cancer therapies. Therefore, we look to animal models of human cancer to help identify key proteins that are physiologically relevant to tumor formation. In this review, I will discuss how scientists are using genetically engineered mice to decipher, at the molecular level, the origins of the pediatric brain tumor, medulloblastoma, and how mouse models are used to design novel therapeutic strategies.

Medulloblastoma is the most common solid tumor afflicting children, and the need for improved treatments for patients with this disease is pressing. Current treatment regimens, which combine surgery with craniospinal radiation

Abbreviations used in this paper: EGL = external granule layer; $\mathrm{GFAP}=$ glial fibrillary acidic protein; $\mathrm{GNP}=$ granule neuron precursor; IFN = interferon; IGF-I and -II = insulin-like growth factor-I and -II; IGF-IR = IGF-I receptor; IRS-1 = insulin receptor substrate -1 ; mRNA = messenger RNA; mTOR = mammalian target of rapamycin; PI3K = phosphatidylinositol 3-kinase; $\mathrm{PTCH}=$ human homolog of the Patched gene; $\mathrm{Rb}=$ retinoblastoma; $\mathrm{Shh}=$ Sonic hedgehog. and multiagent chemotherapy, offer 5-year survival rates of $75 \%$ and result in cures in half of newly diagnosed patients. ${ }^{42}$ Nevertheless, long-term survivors experience skeletal growth retardation, endocrine dysfunction, and progressive cognitive impairment. ${ }^{43,57}$ They are also at increased risk for psychiatric disturbances and social difficulties. ${ }^{52}$ These devastating side effects are attributed to collateral damage to the developing nervous system by anticancer agents, particularly radiation, rather than to the disease itself. Thus, the central practical objective of modern medulloblastoma research is to find ways of increasing treatment specificity for cancer cells and thereby to minimize damage to the developing brain.

What can experiments in mice tell us about human cancer? First, the mouse models discussed in this review have made it possible to determine which cell signaling pathways are required to initiate formation of the tumor and which ones are needed to maintain its growth. Knowing which signaling pathways are activated in mouse tumors can reveal molecular targets for therapeutic intervention in humans, in addition to providing insights into tumor origins. Second, mouse models of human cancer are valuable experimental systems for preclinical testing of novel therapies. Their utility includes testing new chemotherapy drugs and novel uses of existing drugs or combined regimens. Third, mouse models generate an abundant supply of tumor specimens for molecular analysis. This is particularly important for comparatively uncommon types of tumors like 
medulloblastomas. Finally, mouse models are facilitating the development of sensitive and noninvasive imaging modalities.

\section{Expressing and Silencing Genes in Genetically Engineered Mice}

To identify genes that play key roles in medulloblastoma formation, cancer geneticists have used two general classes of genetically engineered mice: transgenic and knock-out mice. Transgenic mice carry an exogenous gene (transgene) with attached control sequences (promoter/enhancer elements) in every cell, both germ cells and somatic cells (Fig. 1A). The promoter/enhancer elements determine the range of tissues and cell types in which the exogenous gene is expressed as well as the developmental timing of gene expression. In transgenic mice, the transgene is overexpressed compared with normal, endogenous genes because of the activity of the attached promoter/enhancer sequences.

In knock-out mice, by contrast, expression of a gene is silenced by replacing an endogenous gene (target gene) with a defective version (Fig. 1B). If both copies (homo$\operatorname{logs}$ ) of the target gene are knocked out, the mice are said to be homozygous defective for the target gene. If only one homolog is defective, the mice are heterozygous defective. If the target gene plays a vital role in embryonic development, then knocking out that gene in the germ line may cause fetal death. Because embryonic lethality is usually uninformative to the mouse geneticist, an alternative strategy has been developed to inactivate a gene at a later point in embryonic development, after it has played its essential role in embryogenesis. Such mice are called conditional knock-outs.

The genetic engineering required to create a conditional knock-out mouse involves flanking the target gene with a set of DNA sequences (LoxP sites; Fig. 1C). These sites are recognized by a bacterial enzyme called Cre recombinase, which excises all DNA sequences interposed between two LoxP sites. In conditional knock-out mice, Cre, the gene encoding Cre recombinase, is expressed as a transgene under control of a promoter/enhancer that is active only in certain cell or tissue types and only at specific times during embryonic development. These conditional knock-out mice, then, contain two transgenes: 1) the target gene, whose expression will be knocked out; and 2) Cre, whose expression is driven by a tissue-specific promoter/enhancer. Thus, when the Cre promoter is switched on in a cell-type-specific manner, the encoded recombinase enzyme removes the target gene through its flanking LoxP sites.

When using genetically engineered mice to design a model of medulloblastoma, one must answer two questions. First, what is the presumed cell of medulloblastoma origin? Second, what signal transduction pathways must be activated in those cells to initiate tumor formation? A substantial body of experimental evidence implicates the GNP as the cell of origin for medulloblastoma. ${ }^{38,64}$ During embryogenesis, undifferentiated GNPs originate from a hindbrain structure called the rhombic lip. A stream of GNPs migrates away from the rhombic lip to the cortical surface of the cerebellum, where these precursors form a secondary germinal zone called the EGL. In mice and in humans, GNPs undergo a rapid postnatal expansion phase, after which they migrate inward, where they differentiate to generate the abundant granule neurons of the internal granule layer. ${ }^{26}$ The rapid proliferation of GNPs during the postnatal expansion phase of the EGL presents a vulnerable setting for transformation events leading to medulloblastoma formation.

\section{Germ Line Inactivation of the Patched Gene Causes Medulloblastoma}

In an attempt to create mouse models that accurately recapitulate the origin of human medulloblastoma, scientists have endeavored to activate signaling pathways that function normally to stimulate proliferation and inhibit differentiation of GNPs. Considerable attention has been focused on the role of the Shh/Patched signaling pathway in the genesis of medulloblastoma (Fig. 2A). Sonic hedgehog is a secreted protein that is a crucial determinant of embryonic pattern formation in the central nervous system; Shh binds the transmembrane protein, Patched, at the cell surface. This interaction activates a signal transduction pathway, leading to transcription of genes that govern various aspects of neural development. ${ }^{28}$ The Gli transcription factors are the terminal effectors of Shh/Patched signaling. In the developing cerebellum, Shh stimulates the proliferation of GNPs and blocks their differentiation into neurons. ${ }^{14,61,65}$

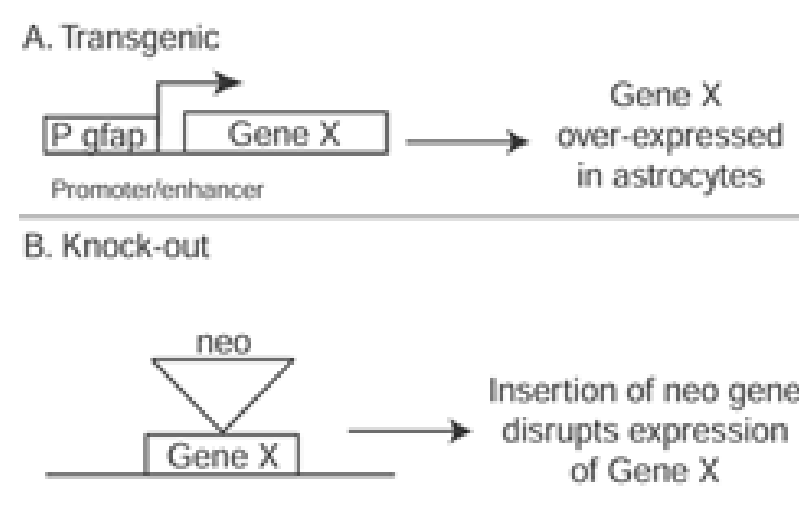

C. Conditional Knock-out

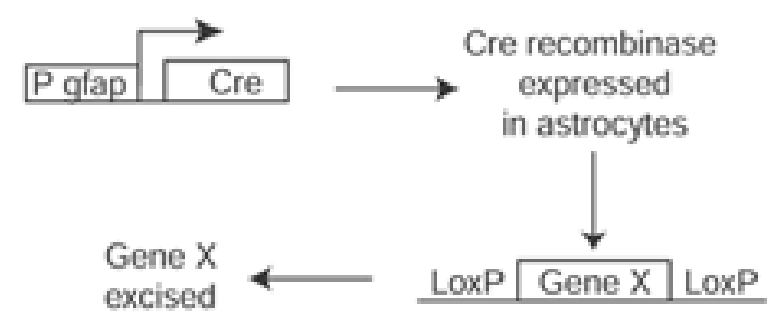

Fig. 1. Schematic drawings showing protocols for using genetically engineered mice to express or knock out a gene in a specific cell type. A: In transgenic mice, a tissue-specific promoter/enhancer is used to drive expression of the transgene (Gene $X$ ). In the example shown, promoter/enhancer sequences from the gene encoding GFAP (gfap) are used to overexpress Gene $X$ in astrocytes. B: In knock-out mice, a sequence (for example, the gene conferrin neomycin resistance gene) is inserted into a target gene (Gene $X$ ) to disrupt expression of the latter. The gene knock-out cassette is propagated from the germ line to all cells of the body. C: In conditional knock-out mice, the target gene (Gene $X$ ) is deleted only in specific cell types (for example, astrocytes). The promoter/enhancer for the GFAP gene is used to drive expression of Cre recombinase, which recognizes LoxP sites and excises Gene $X$ positioned between these sites. 
The first clue that hyperactive $\mathrm{Shh} /$ Patched signaling might play a causal role in human medulloblastoma formation came from an analysis of a rare, hereditary condition called Gorlin syndrome, characterized by neural tube defects, craniofacial abnormalities, and predisposition to various neoplasms, including medulloblastomas. ${ }^{19}$ Genetic analysis revealed that inherited mutations in $P T C H$ segregate in families with Gorlin syndrome. ${ }^{21,30}$ In a subsequent analysis of sporadic medulloblastomas in patients with these lesions, somatically acquired loss-of-function mutations in PTCH were discovered in 3 to $14 \%$ of cases. ${ }^{44,46,70}$ In addition, a hereditary form of medulloblastoma is caused by germ line mutations in the $S U F U$ gene, which encodes a suppressor of the Shh/Patched signaling pathway. ${ }^{59}$ Taken together, these findings have implicated $P T C H$ as a tumor suppressor gene, inactivation of which could promote medulloblastoma formation by activating the Shh/Patched signaling pathway during cerebellar development.

Definitive proof that Patched was a medulloblastoma suppressor gene came from the creation of Patched knock-out mice. In mice that are homozygous defective for Patched, severe defects develop in the neural tube, heart, and other organs, and the animals die at 9 to 10 days of gestation. ${ }^{18,23}$ Heterozygous defective mice are viable, however, and in approximately $14 \%$ aggressive cerebellar tumors develop that closely resemble human medulloblastomas. ${ }^{18,68}$

\section{Activation of the Shh/Patched Signaling Pathway in Developing Cerebellum Induces Medulloblastomas}

The fact that knocking out the Patched gene, which encodes the inhibitory component of the Shh/Patched signaling pathway, induces medulloblastoma predicts that overexpression of Shh itself or a downstream, positive effector of the pathway would also induce tumor formation. Indeed, two experimental strategies in which retroviruses are used to transfer and express Shh in the developing cerebellum induce medulloblastomas. One team of investigators used an ultrasound-guided microinjection technique to transfer an Shh-expressing murine leukemia retrovirus to the developing cerebellum in utero on embryonic Day 13.5. ${ }^{66}$ Medulloblastomas appeared in $76 \%$ of animals, indicating that ectopic expression of Shh is sufficient for medulloblastoma induction. Interestingly, in utero transfer of Shh was still capable of inducing medulloblastomas in mice that were homozygous defective for the Glil gene, which encodes a positively responding, downstream component of the Shh/ Patched signaling pathway.

Another group of investigators used an avian retroviral vector to transfer and express Shh to nestin-expressing neural progenitor cells in the cerebella of newborn mice. They found that ectopic expression of Shh induced tumors in $9 \%$ of mice and that tumor induction increased to $23 \%$ by coexpression of the oncogenic transcription factor c-Myc. ${ }^{47}$ The c-Myc gene is overexpressed in human medulloblastomas, ${ }^{8}$ and accumulation of c-Myc mRNA is an unfavorable prognostic indicator for patients with medulloblastoma. ${ }^{20,27}$

Further evidence that activation of the Shh/Patched signaling pathway induces medulloblastoma was provided by scientists who created a transgenic mouse in which an activated version of the Smoothened gene was expressed in the developing cerebellum. ${ }^{24}$ In this mouse model, transgene expression was driven by promoter/enhancer elements from the NeuroD2 gene, which is expressed at high levels during normal cerebellar development in GNPs within the EGL. Cerebellar tumors closely resembling human medulloblastomas were found in $48 \%$ of mice at a median age of 25.7 weeks.

The Smoothened gene encodes a transmembrane protein that acts as a transducer of extracellular input signals from Shh. The current paradigm is that Patched prevents Smoothened from relaying Shh signals to the interior of the cell and that binding of Shh to Patched stops Patched-mediated repression of Smoothened. The precise molecular mechanism whereby this interaction takes place remains the subject of intense scientific inquiry (see review by $\mathrm{Cohen}^{13}$ ). The Smoothened allele used in the transgenic mouse described

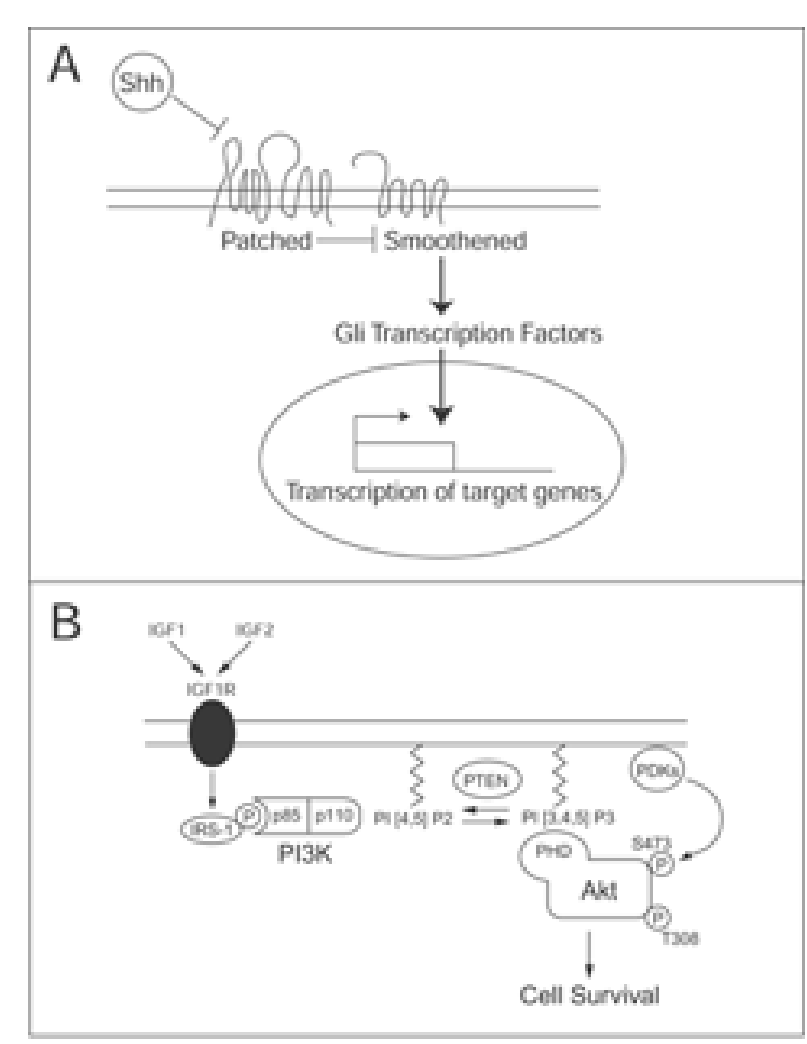

Fig. 2. Schematic drawings showing cell signaling pathways activated in medulloblastoma. A: The Shh/Patched signaling pathway is activated in the medulloblastoma. In the absence of Shh, Patched inhibits transmembrane protein, Smoothened. When Shh binds Patched, this inhibition is relieved. The intermediary reactions leading from ligand-receptor (Shh-Patched) binding at the cell surface to transcription activation in the nucleus are not completely understood. The effect of Shh is to activate the Gli family of transcription factors, which stimulate transcription of target genes. B: The IGFs signal through the PI3K pathway. Engagement of IGF-IR (IGF1R) by IGF-I or IGF-II activates the receptor tyrosine kinase. Phosphorylation of IRS-1 by IGF-IR creates a docking site for the regulatory subunit (p85) of PI3K. Consequent activation of the catalytic subunit (p110) generates phosphatidylinositol 3,4,5-trisphosphate (PI [3,4,5] P3), which recruits Akt to the plasma membrane through Akt's pleckstrin homology domain (PHD). Serine/threonine phosphorylation of Akt at the plasma membrane by 3-phosphoinositide-dependent kinases (PDKs) activates Akt. The PI3K signaling is suppressed by the phosphatase activity of tumor suppressor protein, PTEN. 
earlier encodes a mutant protein with a single amino acid substitution (leucine substituted for the normal tryptophan at position 535). Structure-function studies suggest that this missense mutant of Smoothened is constitutively active, in that it continuously transduces an Shh-like signal, even in the absence of Shh. Missense mutations in the Smoothened gene occur in human neuroectodermal tumors, albeit rarely. ${ }^{49,72}$ Taken together, these studies indicate that activation of the Shh/Patched signaling pathway in the developing cerebellum is sufficient to initiate medulloblastoma formation and that undifferentiated neural progenitor cells, which are present in the cerebellum at birth, can act as the cells of origin for medulloblastoma.

\section{Hyperplasia of the EGL: a Precursor Stage of Medulloblastoma}

In the mouse models discussed earlier, in which medulloblastomas were induced by ectopic expression of Shh or Smoothened, examination of the cerebellar cortex showed abnormal thickening of the EGL, even in mice with no obvious tumors. ${ }^{24,47,66}$ This observation suggests that EGL hyperplasia may be a precursor stage of medulloblastoma. In support of this concept, foci of GNP-like cells have been identified on the cortical surface of the cerebella from Patched-deficient mice at time points in development after GNPs have normally migrated inward from the EGL to the internal granule layer. ${ }^{41}$ Isolation and molecular characterization revealed that these cells have activation of the $\mathrm{Shh} /$ Patched signaling pathway and that they proliferate in culture. Transcriptional profiling using DNA microarrays showed a gene expression pattern distinct from that of GNPs obtained in wild-type mice, suggesting that these cells mark a preneoplastic stage of medulloblastoma.

\section{Overproduction of Immune Response Proteins can Initiate} Medulloblastoma Formation

If overstimulation of GNPs by Shh is the proximate cause of medulloblastoma formation, then what environmental or developmental cues might trigger this process? The human neurotropic polyomavirus, JC virus, has been implicated as a cause of medulloblastoma because $\mathrm{JC}$ virus antigens are present in human medulloblastomas and because transgenic mice expressing the JC virus $\mathrm{T}$ antigen develop medulloblastomas. ${ }^{63}$ Virus-induced carcinogenesis is not the topic of this review. Nevertheless, two transgenic mouse models have shown that IFNs, a class of cytokines that mediate a host's response to viral infection, can induce medulloblastoma formation when overexpressed in the developing brain. One team of investigators created a transgenic mouse in which IFN- $\alpha$ was expressed in the central nervous system via promoter/enhancer elements from the GFAP gene. ${ }^{37}$ Highly aggressive medulloblastomas developed in these mice, and nearly $100 \%$ of the animals died by 1 month after birth. Tumors developed only in mice in which the gene encoding signal transducer and activator of transcription 2 was knocked out. Signal transducer and activator of transcription proteins are intracellular signaling molecules that become activated when cytokines like IFN$\alpha$ engage their cognate receptors on the cell surface. Analysis of the tumors induced by IFN- $\alpha$ revealed that the Shh/ Patched signaling pathway was activated (through a signal transducer and activator of transcription 1-dependent mechanism), consistent with the idea that $S h h$ plays an essential role in medulloblastoma formation. Furthermore, the authors showed that Shh/Patched pathway activation was a direct response to a different IFN, IFN- $\gamma$, which was produced by $\mathrm{T}$ lymphocytes migrating into the brain in response to IFN- $\alpha$.

These results suggested that T-cell-derived IFN- $\gamma$ was capable of initiating medulloblastoma formation if present in sufficient quantity and during the appropriate window of cerebellar development. Direct proof for this concept was provided by Lin, et al., ${ }^{37}$ who created a transgenic mouse in which IFN- $\gamma$ was expressed in the developing nervous system under control of the GFAP gene promoter. Through genetic engineering, the investigators were able to control the timing of IFN- $\alpha$ transgene expression. Medulloblastomas developed in $80 \%$ of mice in which IFN- $\gamma$ was expressed at embryonic Day 16, but no tumors developed when IFN- $\alpha$ expression was switched on at birth. As in the IFN- $\alpha$ model, induction of medulloblastomas by IFN- $\gamma$ correlated with activation of the Shh/Patched signaling pathway. Although IFN- $\gamma$ was necessary for medulloblastoma initiation in this mouse model, continued IFN- $\gamma$ expression was not required for tumor progression. Taken together, these two mouse models raise the intriguing possibility that increased expression of IFNs by viral infection in the cerebellum might be an inciting event that activates Shh/Patched signaling, thus leading to medulloblastoma formation.

\section{Deficiency of $\mathrm{p} 53$ Cooperates With Shh to Accelerate Medulloblastoma Formation in Mice}

Although the observations discussed earlier strongly implicate Shh/Patched signaling as a causative factor for medulloblastoma, mounting evidence indicates that this pathway is not the sole determinant of medulloblastoma formation. First, the frequency of mutations in Shh/Patched pathway genes in human medulloblastomas is very low. ${ }^{72}$ Second, medulloblastomas arise in only $14 \%$ of Patcheddeficient mice within the 1 st year of life, ${ }^{68}$ and tumor incidence is highly dependent on the genetic background of the knock-out mice. ${ }^{9}$ What other molecular signals could subvert the normal process of neural differentiation in the cerebellum and bring about neoplastic transformation?

Medulloblastoma formation is dramatically accelerated in double knock-out mice that are not only heterozygous defective for Patched (Patched ${ }^{+/}$) but also homozygous defective for the $p 53$ gene $\left(p 53^{-/-}\right) \cdot{ }^{67} \mathrm{~A}$ well-known tumor suppressor gene, $p 53$ encodes a transcription factor that inhibits cell cycle progression and mediates cellular response to DNA damage by triggering apoptosis. ${ }^{25}$ More than $95 \%$ of Patched ${ }^{+-} / p 53^{-1-}$ mice had tumors at 10 to 12 weeks of age, compared with a $14 \%$ incidence in Patched ${ }^{+1-}$ mice at 10 months. The fact that medulloblastomas from Patched $d^{+/-} / p 53^{-/}$mice showed a higher rate of chromosome loss on cytogenetic analysis than tumors from Patched ${ }^{+/}$mice suggests that the effect of p53 deficiency on tumor induction might be related to genomic instability and acquisition of secondary mutations in growth control genes. Despite the impact of p53 deficiency on medulloblastoma formation in genetically engineered mice, loss-of-function mutations in the $p 53$ gene are rare in human medulloblastomas. ${ }^{1,3,54}$ 
Modeling medulloblastoma with genetically engineered mice

\section{Defective DNA Repair Programs Promote Medulloblastoma Formation}

The high rate of chromosome loss in tumor cells from Patched $^{+-} / \mathrm{P} 3^{--}$mice points to genomic instability as a driving force in medulloblastoma formation. This idea is supported by two other mouse models of medulloblastoma in which important genes governing DNA repair were knocked out. One mouse was a double knock-out of p53 and Lig4, the gene encoding DNA ligase IV, an enzyme that is critical for repairing double-strand DNA breaks. ${ }^{35}$ The second mouse model was created by knocking out p53 in combination with the gene encoding poly(adenosine $5^{\prime}$-diphosphate-ribose) polymerase, a molecule that senses DNA strand breaks. Activation of poly(adenosine 5'-diphosphate-ribose) polymerase is an early response to DNA damage. ${ }^{60}$ The $_{\text {Lig4 }}{ }^{-1-}$ mice die in early embryogenesis because of widespread neural apoptosis. ${ }^{34}$ Additional loss of p53 rescues normal neural development in Lig4 $^{-1-}$ mice, but it predisposes them to tumorigenesis. Loss of p53 may be required to generate medulloblastomas in mice with defective DNA repair systems because p53 functions normally to arrest cell cycle progression after DNA damage. In the absence of this control mechanism, DNA strand breaks in genes controlling cell growth are perpetuated because damaged cells are not eliminated via apoptosis.

\section{Activation of the PI3K Signaling Pathway by IGF-II Synergizes With Shh to Enhance Medulloblastoma Formation}

Molecular oncology studies in humans and mice indicate that cell survival signals from IGFs are additional, causative factors for medulloblastoma. For example, transcriptional profiling of human medulloblastomas by using DNA microarrays has shown increased expression of IGF-II. ${ }^{45} \mathrm{Ho}-$ mozygous deletion of the $I g f 2$ gene completely eliminates medulloblastoma formation in Patched $^{-/-}$mice, suggesting a requirement for IGF-II or downstream signaling molecules in Shh-induced medulloblastoma formation. ${ }^{22}$

The physiological effects of IGF-II are mediated by IGFIR, a receptor tyrosine kinase, activation of which triggers an important signal transduction pathway known as the PI3K pathway. ${ }^{33}$ Salient features of the PI3K signaling pathway are illustrated schematically in Fig. 2B. In brief, phosphorylation of IRS-1 by activated IGF-IR creates a docking site for the regulatory subunit of PI3K. Consequent activation of the catalytic subunit of PI3K generates the membrane phospholipid, phosphatidylinositol (3,4,5)-trisphosphate, which recruits the serine/threonine kinase Akt (protein kinase B) to the plasma membrane, where phosphorylation of this molecule activates the Akt kinase. ${ }^{10}$

The first clues that IGF-mediated PI3K signaling might play a role in medulloblastoma formation came from studies of medulloblastoma cell lines derived from transgenic mice expressing JC virus $\mathrm{T}$ antigen. ${ }^{62}$ These cell lines showed increased expression of IRS-1, and exogenous IGFI stimulated them to proliferate in culture. Furthermore, immunohistochemical studies of primary human medulloblastomas have shown expression of IRS-1 and phosphorylated (activated) IGF-IR. ${ }^{15}$

To evaluate the role of IGF signaling in medulloblastoma formation in vivo, we used the RCAS/ $t v-a$ mouse system, which allows postnatal gene transfer in a cell-type-specific manner. ${ }^{16,29}$ The RCAS/ $t v-a$ system (Fig. 3) uses an avian retroviral vector, called RCAS, derived from avian leukosis virus, ALV (subgroup A), and a transgenic mouse line $(N t v-a)$ that produces TV-A (the receptor for ALV-A) under control of the Nestin gene promoter. Nestin is an intermediate filament protein expressed by neuronal and glial progenitor cells. ${ }^{36}$ When RCAS retroviruses infect mammalian cells, virus replication does not occur. Instead, retroviral RNA is reverse-transcribed to generate a complementary copy of DNA (proviral DNA), which then integrates into the host cell genome. The integrated gene is expressed as a spliced message under control of the constitutive retroviral promoter LTR, or long terminal repeat. This system makes it possible to transfer and express exogenous genes in nestin-expressing neural progenitor cells (the presumed cells of origin for medulloblastoma) inside the brains of live mice. Furthermore, combinations of genes can be transferred to individual cells by infection with multiple RCAS vectors carrying different genes. This makes it possible to determine whether different oncogenes cooperate to induce tumor formation in vivo.

Using the RCAS/ $t v-a$ system, we transferred genes encoding IGF-II and Shh, alone and in combination, to nestinexpressing neural progenitors by injecting virus producer cells into the cerebella of newborn Ntv-a mice. Combined expression of IGF-II + Shh induced medulloblastomas in $39 \%$ of mice, a significantly higher incidence than in those with Shh alone, which induced tumors in 9 to $15 \%$ of animals. ${ }^{48}$ Tumors induced by Shh or Shh + IGF-II showed increased expression of activated IGF-I receptor as well as downstream signaling proteins, IRS-1 and Akt. This finding suggests that sustained IGF signaling is required to maintain growth of tumors once their development is initiated by Shh.

To demonstrate a functional role for PI3K signaling in medulloblastoma formation, we transferred an activated, transforming allele of $A k t$. We found that Akt had an enhancing effect on Shh-induced medulloblastoma formation comparable to that of IGF-II (48\% tumor incidence). ${ }^{48} \mathrm{Nev}$ ertheless, neither IGF-II nor Akt was sufficient for tumor induction when expressed independently. These results with modeling medulloblastoma by using the RCAS/ $t v-a$ system indicate that combined activation of the Shh/Patched and IGF/PI3K signaling pathways is an important oncogenic mechanism leading to medulloblastoma formation.

\section{Loss of Cell Cycle Control: a Driving Force in Medulloblastoma Oncogenesis}

Mouse models of medulloblastoma have shown that an essential ingredient for tumor formation is a signal that drives GNPs through the cell cycle. The presence of Shh stimulates progression of GNPs through the $G_{1}$ phase of the cell cycle by increased expression of D-type cyclins, phosphorylation (and hence inactivation) of the $\mathrm{Rb}$ gene product, and activation of E2F transcription factors. ${ }^{32} \mathrm{Di}$ rect evidence for the role of $\mathrm{Rb}$ signaling in the genesis of medulloblastoma came from conditional knock-out of the $R b$ gene in mice that were homozygous defective for $p 53 .{ }^{39}$ To generate these mice, the investigators drove expression of Cre recombinase via the promoter for GFAP, which is normally expressed in mature astrocytes. The fact that tumor formation and GFAP-driven expression of the Cre 


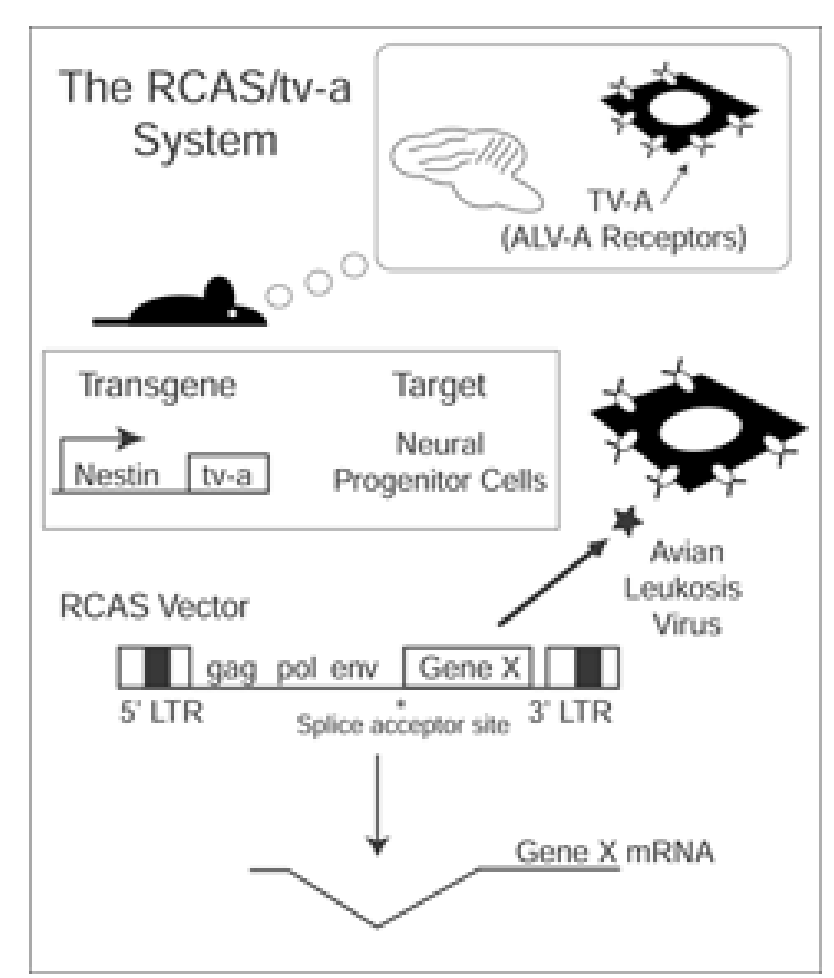

Fig. 3. Schematic drawings showing the RCAS/tv- $a$ system. The ALV receptors (TV-A) are produced in nestin-expressing neural progenitor cells in Ntv-a transgenic mice. The RCAS vectors carrying an exogenous gene (Gene $X$ ) replicate in avian cells to produce live virus capable of infecting $\mathrm{TV}-\mathrm{A}^{+}$mouse cells. Virus production occurs only in avian cells. In mouse cells, the exogenous gene is expressed as a spliced message from which viral genes (gag, pol, env) are removed. LTR = long terminal repeat.

transgene both occurred in the EGL in these mice further supports the idea that GNPs are the cells of origin for medulloblastoma. Conditional inactivation of $R b$ was not sufficient, however, to induce tumors in mice with normal levels of p53. Cooperative effects of $\mathrm{Rb}$ and $\mathrm{p} 53$ signaling on central nervous system tumor formation were also demonstrated by an earlier, transgenic mouse model expressing SV40 large T antigen, which functionally inactivates both $\mathrm{Rb}$ and $\mathrm{p} 53$. Primitive neuroectodermal tumors developed in the brainstem in these mice. ${ }^{17}$

Another set of cell cycle control proteins that plays an inhibitory role in medulloblastoma formation is the Ink4 family (p16 ${ }^{\text {Ink4a }}, \mathrm{p} 15^{\text {Ink4b }}, \mathrm{p} 18^{\text {Ink4c }}$, and $\mathrm{p} 19^{\text {Ink4d }}$ ). These proteins normally prevent cells from entering the DNA synthetic phase (S phase) of the cell cycle. ${ }^{56}$ They do this by blocking phosphorylation of the $\mathrm{Rb}$ protein, thereby maintaining it in its active, growth-inhibitory state. The important role of Ink4a proteins in restraining cell cycle progression predicts that inactivation of these proteins might promote tumor formation in mice. Indeed, mice that are homozygous defective for both Ink4c and P53 develop medulloblastomas, as well as hemangiosarcomas, lymphomas, and pituitary adenomas. ${ }^{71}$

\section{Mouse Models of Medulloblastoma can Guide Development of Molecular Targeted Therapy}

A discouraging result of molecular cancer research has been the discovery that cancer cells have many defective growth control programs, which makes selecting the best targets for therapeutic intervention an overwhelming task. How can mouse models be exploited to simplify this task? The central role of activated Shh/Patched signaling in the initiation and maintenance of medulloblastoma in mice has exposed a potential Achilles heel of this tumor for therapeutic intervention. Small-molecular-weight compounds that inhibit Shh/Patched signaling have been derived from steroidal alkaloids found in the range plant Veratrum californicum. ${ }^{31}$ Veterinary scientists recognized that this plant might contain compounds capable of perturbing neural development after observing that pregnant ewes that ingested $\mathrm{V}$. californicum gave birth to lambs with holoprosencephaly, a midline craniofacial deformity whose most striking feature is the presence of a single eye (cyclopia). ${ }^{6}$ In humans, loss-offunction mutations in the Shh gene, which would suppress $\mathrm{Shh} /$ Patched pathway activity, cause holoprosencephaly.,50 One of the plant alkaloids, cyclopamine, was subsequently shown to inhibit Smoothened, the positively responding component of the Shh/Patched signaling pathway. ${ }^{12,58}$ When medulloblastoma cells derived from Patched-deficient mice are exposed to cyclopamine in culture, these cells stop proliferating and differentiate into neurons. ${ }^{5}$

Another exciting step toward pharmacological inhibition of Shh/Patched signaling in medulloblastoma treatment was taken by Romer, et al., ${ }^{51}$ who used the Patched ${ }^{+/-}$/ $p 53^{-1-}$ mouse model of medulloblastoma for preclinical testing of a cyclopamine derivative, HhAntag-691. They showed significant reduction in tumor volume and prolonged tumor-free survival in treated animals. The fact that animals receiving even the highest drug doses showed no toxic side effects has raised hopes of using cyclopamine analogs in human clinical trials.

The fact that activation of the PI3K signaling pathway by IGF-II synergizes with Shh to enhance medulloblastoma formation in mice suggests that pharmacological blockade of the PI3K signaling pathway might be a rational therapeutic approach. ${ }^{48}$ For clinicians, this is an exciting prospect because several nodes along the pathway can be selectively blocked by small molecules. ${ }^{11}$ The most strategic target for therapeutic intervention might be mTOR, a serine/threonine kinase, activation of which promotes mRNA translation and hence cell cycle progression. The precise biochemical mechanism whereby PI3K signaling activates mTOR is currently the focus of intense research, and is a topic beyond the scope of this review. Nevertheless, mTOR is a phosphorylation substrate for Akt, and therefore functional interference with mTOR can significantly impede PI3K signaling. ${ }^{40,55}$

Exposure of tumor cells to rapamycin, a macrolide antibiotic drug, markedly suppresses growth of tumor cells in culture by interfering with mTOR's ability to activate mRNA translational machinery. ${ }^{7}$ Rapamycin was first developed as an antifungal drug. It was subsequently found to be a potent immunosuppressor, and this discovery led to approval by the US Food and Drug Administration for the use of rapamycin for preventing rejection of transplanted organs. Rapamycin and several chemical analogs are now in human clinical trials, and evidence is accumulating that they are effective anticancer agents. ${ }^{53}$ For example, measurable response to the Wyeth Ayerst compound CCI-779 was 44 and $7 \%$ in heavily pretreated patients with mantle 
cell lymphoma and renal cell carcinoma, respectively., 2,69 Moreover, Phase I and II cancer trials have shown that these drugs are well tolerated by patients, a surprising result considering the importance of PI3K signaling in maintaining cell growth and survival. Reported toxic effects have been mild, and include cutaneous reactions, transitory myelosuppression, and reversible hepatic dysfunction. Chronic immunosuppression has not been reported with the dose schedules used in current clinical trials. Although mTOR inhibitors have not yet been used in clinical trials for patients with medulloblastoma, the fact that PI3K signaling is frequently activated in mouse and human medulloblastomas, coupled with the need for small-molecular-weight compounds to penetrate the blood-brain barrier and target migratory brain tumor cells, indicates that $\mathrm{mTOR}$ is a rational target for medulloblastoma chemotherapy.

Preclinical testing of mTOR inhibitors in rodent xenograft models of several types of human cancers has shown that delay in tumor growth occurs more commonly than tumor regression. ${ }^{7,53}$ This raises the possibility that, for maximum efficacy, rapamycin analogs should be used in conjunction with cytotoxic chemotherapeutic agents or with radiation therapy. Development of the best combinatorial drug regimens will require mouse models of human tumors that accurately reflect the molecular pathogenesis of human disease.

\section{CONCLUSIONS}

Models of human medulloblastoma in genetically engineered mice have shown that aberrant activation of signal transduction pathways that promote proliferation and inhibit differentiation of neural progenitor cells during normal cerebellar development is the proximate cause of medulloblastoma. The Shh/Patched pathway stands out as the principal mediator of tumor formation, with accessory or permissive roles played by IGF-mediated PI3K signaling, IFNs, Myc oncoproteins, and loss of DNA damage repair mechanisms. Mouse models have revealed molecular targets within the Shh/Patched and PI3K signaling pathways for therapeutic intervention, and these models will remain sturdy platforms for preclinical testing of novel chemotherapy agents.

\section{References}

1. Adesina AM, Nalbantoglu J, Cavenee WK: p53 gene mutation and $M D M 2$ gene amplification are uncommon in medulloblastoma. Cancer Res 54:5649-5651, 1994

2. Atkins MB, Hidalgo M, Stadler WM, et al: Randomized phase II study of multiple dose levels of CCI-779, a novel mammalian target of rapamycin kinase inhibitor, in patients with advanced refractory renal cell carcinoma. J Clin Oncol 22:909-918, 2004

3. Badiali M, Iolascon A, Loda M, et al: $p 53$ gene mutations in medulloblastoma. Immunocytochemistry, gel shift analysis, and sequencing. Diagn Mol Pathol 2:23-28, 1993

4. Belloni E, Muenke M, Roessler E, et al: Identification of Sonic hedgehog as a candidate gene responsible for holoprosencephaly. Nat Genet 14:353-356, 1996

5. Berman DM, Karhadkar SS, Hallahan AR, et al: Medulloblastoma growth inhibition by hedghog pathway blockade. Science 297:1559-1561, 2002

6. Binns W, James LF, Shupe JL, et al: A congenital cyclopian-type malformation in lambs induced by maternal ingestion of a range plant, Veratrum californicum. Am J Vet Res 24:1164-1175, 1963
7. Bjornsti MA, Houghton PJ: The TOR pathway: a target for cancer therapy. Nat Rev Cancer 4:335-348, 2004

8. Bruggers CS, Tai KF, Murdock T, et al: Expression of the cMyc protein in childhood medulloblastoma. J Pediatr Hematol Oncol 20:18-25, 1998

9. Calzada-Wack J, Kappler R, Schnitzbauer TR, et al: Unbalanced overexpression of the mutant allele in murine Patched mutants. Carcinogenesis 23:727-733, 2002

10. Cantley LC: The phosphoinositide 3-kinase pathway. Science 296:1655-1657, 2002

11. Chang F, Lee JT, Navolanic PM, et al: Involvement of PI3K/ Akt pathway in cell cycle progression, apoptosis, and neoplastic transformation: a target for cancer chemotherapy. Leukemia 17:590-603, 2003

12. Chen JK, Taipale J, Cooper MK, et al: Inhibition of Hedgehog signaling by direct binding of cyclopamine to Smoothened. Genes Dev 16:2743-2748, 2002

13. Cohen MM Jr: The hedghog signaling network. Am J Med Genet 123:5-28, 2003

14. Dahmane N, Ruiz-i-Altaba A: Sonic hedgehog regulates the growth and patterning of the cerebellum. Development 126: 3089-3100, 1999

15. Del Valle L, Enam S, Lassak A, et al: Insulin-like growth factor I receptor activity in human medulloblastomas. Clin Cancer Res 8:1822-1830, 2002

16. Federspiel MJ, Bates P, Young JAT, et al: A system for tissuespecific gene targeting: transgenic mice susceptible to subgroup A avian leukosis virus-based retroviral vectors. Proc Nat Acad Sci U S A 91:11241-11245, 1994

17. Fung KM, Trojanowski JQ: Animal models of medulloblastomas and related primitive neuroectodermal tumors: a review. J Neuropathol Exp Neurol 54:285-296, 1995

18. Goodrich LV, Milenkovic L, Higgins KM, et al: Altered neural cell fates and medulloblastoma in mouse patched mutants. Science 277:1109-1113, 1997

19. Gorlin RJ: Nevoid basal cell carcinoma syndrome. Medicine (Baltimore) 66:98-113, 1987

20. Grotzer MA, Hogarty MD, Janss AJ, et al: $M Y C$ messenger RNA expression predicts survival outcome in childhood primitive neuroectodermal tumor/medulloblastoma. Clin Cancer Res 7: 2425-2433, 2001

21. Hahn H, Wicking CA, Zaphiropoulos PG, et al: Mutations of the human homolog of Drosophila patched in the nevoid basal cell carcinoma syndrome. Cell 85:841-851, 1996

22. Hahn H, Wojnowski L, Specht K, et al: Patched target Igf2 is indispensable for the formation of medulloblastoma and rhabdomysosarcoma. J Biol Chem 275:28341-28344, 2000

23. Hahn H, Wojnowski L, Zimmer AM, et al: Rhabdomyosarcomas and radiation hypersensitivity in a mouse model of Gorlin syndrome. Nat Med 4:619-622, 1998

24. Hallahan AR, Pritchard JI, Hansen S, et al: The SmoA1 mouse model reveals that notch signaling is critical for the growth and survival of sonic hedgehog-induced medulloblastomas. Cancer Res 64:7794-7800, 2004

25. Harris SL, Levine AJ: The p53 pathway: positive and negative feedback loops. Oncogene 24:2899-2908, 2005

26. Hatten ME, Heintz N: Mechanisms of neural patterning and specification in the developing cerebellum. Ann Rev Neurosci 18:385-408, 1995

27. Herms J, Neidt I, Luscher B, et al: C-MYC expression in medulloblastoma and its prognostic value. Int J Cancer 89:395-402, 2000

28. Ho KS, Scott MP: Sonic hedgehog in the nervous system: functions, modifications and mechanisms. Curr Opin Neurobiol 12:57-63, 2002

29. Holland EC, Varmus HE: Basic fibroblast growth factor induces cell migration and proliferation after glia-specific gene transfer in mice. Proc Nat Acad Sci U S A 95:1218-1223, 1998

30. Johnson RL, Rothman AO, Xie J, et al: Human homolog of 
patched, a candidate gene for the basal cell nevus syndrome. Science 272:1668-1671, 1996

31. Keeler RF, Binns W: Teratogenic compounds of Veratrum californicum (Durand). V. Comparison of cyclopian effects of steroidal alkaloids from the plant and structurally related compounds from other sources. Teratology 1:5-10, 1968

32. Kenney AM, Rowitch DH: Sonic hedgehog promotes $\mathrm{G}_{1}$ cyclin expression and sustained cell cycle progression in mammalian neuronal precursors. Mol Cell Biol 20:9055-9067, 2000

33. Khandwala HM, McCutcheon IE, Flyvbjerg A, et al: The effects of insulin-like growth factors on tumorigenesis and neoplastic growth. Endocr Rev 21:215-244, 2000

34. Lee Y, Barnes DE, Lindahl T, et al: Defective neurogenesis resulting from DNA ligase IV deficiency requires Atm. Genes Dev 14:2576-2580, 2000

35. Lee Y, McKinnon PJ: DNA ligase IV suppresses medulloblastoma formation. Cancer Res 62:6395-6399, 2002

36. Lendahl U, Zimmerman LB, McKay RDG: CNS stem cells express a new class of intermediate filament protein. Cell 60: 585-595, 1990

37. Lin W, Kemper A, McCarthy KD, et al: Interferon-gamma induced medulloblastoma in the developing cerebellum. J Neurosci 24:10074-10083, 2004

38. Marino S: Medulloblastoma: developmental mechanisms out of control. Trends Mol Med 11:17-22, 2005

39. Marino S, Vooijs M, van Der Gulden H, et al: Induction of medulloblastomas in p53-null mutant mice by somatic inactivation of $R b$ in the external granular layer cells of the cerebellum. Genes Dev 14:994-1004, 2000

40. Navé BT, Ouwens M, Withers DJ, et al: Mammalian target of rapamycin is a direct target for protein kinase B: identification of a convergence point for opposing effects of insulin and aminoacid deficiency on protein translation. Biochem J 344:427-431, 1999

41. Oliver TG, Read TA, Kessler JD, et al: Loss of patched and disruption of granule cell development in a pre-neoplastic stage of medulloblastoma. Development 132:2425-2439, 2005

42. Packer RJ, Cogen P, Vezina G, et al: Medulloblastoma: clinical and biologic aspects. Neuro-oncol 1:232-250, 1999

43. Packer RJ, Sutton LN, Atkins TE, et al: A prospective study of cognitive function in children receiving whole-brain radiotherapy and chemotherapy: 2-year results. J Neurosurg 70:707-713, 1989

44. Pietsch T, Waha A, Koch A, et al: Medulloblastomas of the desmoplastic variant carry mutations of the human homologue of Drosophila patched. Cancer Res 57:2085-2088, 1997

45. Pomeroy SL, Tamayo P, Gaasenbeek M, et al: Prediction of central nervous system embryonal tumor outcome based on gene expression. Nature 415:436-442, 2002

46. Raffel C, Jenkins RB, Frederick L, et al: Sporadic medulloblastomas contain PTCH mutations. Cancer Res 57:842-845, 1997

47. Rao G, Pedone CA, Coffin CM, et al: c-Myc enhances Sonic hedgehog-induced medulloblastoma formation from nestin-expressing neural progenitors in mice. Neoplasia 5:198-204, 2003

48. Rao G, Pedone CA, Del Valle L, et al: Sonic hedgehog and insulin-like growth factor signaling synergize to induce medulloblastoma formation from nestin-expressing neural progenitors in mice. Oncogene 23:6156-6162, 2004

49. Reifenberger J, Wolter M, Weber RG, et al: Missense mutations in $\mathrm{SMOH}$ in sporadic basal cell carcinomas of the skin and primitive neuroectodermal tumors of the central nervous system. Cancer Res 58:1798-1803, 1998

50. Roessler E, Belloni E, Gaudenz K, et al: Mutations in the human Sonic Hedgehog gene cause holoprosencephaly. Nat Genet 14:357-360, 1996

51. Romer JT, Kimura H, Magdaleno S, et al: Suppression of the Shh pathway using a small molecule inhibitor eliminates medulloblastoma in $\mathrm{Ptc}^{+/-}$p53-1- mice. Cancer Cell 6:229-240, 2004

52. Ross L, Johansen C, Dalton SO, et al: Psychiatric hospitaliza- tions among survivors of cancer in childhood or adolescence. $\mathbf{N}$ Engl J Med 349:650-657, 2003

53. Rowinsky EK: Targeting the molecular target of rapamycin (mTOR). Curr Opin Oncol 16:564-575, 2004

54. Saylors RL II, Sidransky D, Friedman HS, et al: Infrequent p53 mutations in medulloblastomas. Cancer Res 51:4721-4723, 1991

55. Scott PH, Brunn GJ, Kohn AD, et al: Evidence of insulin-stimulated phosphorylation and activation of the mammalian target of rapamycin mediated by a protein kinase B signaling pathway. Proc Natl Acad Sci U S A 95:7772-7777, 1998

56. Sherr CJ, Roberts JM: Living with or without cyclins and cyclin-dependent kinases. Genes Dev 18:2699-2711, 2004

57. Silber JH, Littman PS, Meadows AT: Stature loss following skeletal irradiation for childhood cancer. J Clin Oncol 8:304-312, 1990

58. Taipale J, Chen JK, Cooper MK, et al: Effects of oncogenic mutations in Smoothened and Patched can be reversed by cyclopamine. Nature 406:1005-1009, 2000

59. Taylor MD, Liu L, Raffel C, et al: Mutations in $S U F U$ predispose to medulloblastoma. Nat Genet 31:306-310, 2002

60. Tong WM, Ohgaki H, Huang $\mathrm{H}$, et al: Null mutation of DNA strand break-binding molecule poly(ADP-ribose) polymerase causes medulloblastomas in $\mathrm{p} 53^{-/-}$mice. Am J Pathol 162: 343-352, 2003

61. Wallace VA: Purkinje-cell-derived Sonic hedgehog regulates granule neuron precursor cell proliferation in the developing mouse cerebellum. Curr Biol 9:445-448, 1999

62. Wang JY, Del Valle L, Gordon J, et al: Activation of the IGF-1R system contributes to malignant growth of human and mouse medulloblastomas. Oncogene 20:3857-3868, 2001

63. Wang JY, Del Valle L, Peruzzi F, et al: Polyomaviruses and cancer-interplay between viral proteins and signal transduction pathways. J Exp Clin Cancer Res 23:373-383, 2004

64. Wechsler-Reya R, Scott MP: The developmental biology of brain tumors. Ann Rev Neurosci 24:385-428, 2001

65. Wechsler-Reya RJ, Scott MP: Control of neuronal precursor proliferation in the cerebellum by Sonic Hedgehog. Neuron 22:103-114, 1999

66. Weiner HL, Bakst R, Hurlbert MS, et al: Induction of medulloblastomas in mice by Sonic hedgehog, independent of Gli1. Cancer Res 62:6385-6389, 2002

67. Wetmore C, Eberhart DE, Curran T: Loss of $p 53$ but not $A R F$ accelerates medulloblastoma in mice heterozygous for patched. Cancer Res 61:513-516, 2001

68. Wetmore C, Eberhart DE, Curran T: The normal patched allele is expressed in medulloblastomas from mice with heterozygous germ-line mutation of patched. Cancer Res 60:2239-2246, 2000

69. Witzig TE, Geyer SM, Ghobrial I, et al: Phase II trial of singleagent temsirolimus (CCI-779) for relapsed mantle cell lymphoma. J Clin Oncol 23:5347-5356, 2005

70. Wolter M, Reifenberger J, Sommer C, et al: Mutations in the human homologue of the Drosophila segment polarity gene patched $(P T C H)$ in sporadic basal cell carcinomas of the skin and primitive neuroectodermal tumors of the central nervous system. Cancer Res 57:2581-2585, 1997

71. Zindy F, Nilsson LM, Nguyen L, et al: Hemangiosarcomas, medulloblastomas, and other tumors in Ink4c/p53-null mice. Cancer Res 63:5420-5427, 2003

72. Zurawel RH, Allen C, Chiappa S, et al: Analysis of PTCH/ SMO/SHH pathway genes in medulloblastoma. Genes Chromosomes Cancer 27:44-51, 2000

Manuscript received September 15, 2005

Accepted in final form October 17, 2005

Address reprint requests to: Daniel W. Fults, M.D., Department of Neurosurgery, University of Utah School of Medicine, 30 North 1900 East, Salt Lake City, Utah 84132-2303. email: daniel.fults@ hsc.utah.edu. 